\title{
The Periplasmic Chaperone Skp Facilitates Targeting, Insertion, and Folding of OmpA into Lipid Membranes with a Negative Membrane Surface Potential ${ }^{\dagger}$
}

\author{
Geetika J. Patel, ${ }^{\ddagger}$ Susanne Behrens-Kneip, ${ }^{\S}$ Otto Holst,, and Jörg H. Kleinschmidt* ${ }^{*}$ \\ ${ }^{\sharp}$ Fachbereich Biologie, Universität Konstanz, Universitätsstraße 10, D-78464 Konstanz, Germany, ${ }^{\S}$ Robert-Koch-Institute, \\ P26 Nosocomial Infections of the Elderly, Nordufer 20, 13353 Berlin, Germany, and Division of Structural Biochemistry, \\ Research Center Borstel, Leibniz-Center for Medicine and Biosciences, Parkallee 4a/c, D-23845 Borstel, Germany
}

Received August 12, 2009; Revised Manuscript Received September 24, 2009

\begin{abstract}
The basic biochemical and biophysical principles by which chaperone-bound membrane proteins are targeted to the outer membrane of Gram-negative bacteria for insertion and folding are unknown. Here we compare spontaneous folding of outer membrane protein A (OmpA) of Escherichia coli from its ureaunfolded form and from the complex with its periplasmic chaperone Skp into lipid bilayers. Skp facilitated folding of OmpA into negatively charged membranes containing dioleoylphosphatidylglycerol (DOPG). In contrast, Skp strongly inhibited folding of OmpA when bilayers were composed of dioleoylphosphatidylethanolamine and dioleoylphosphatidylcholine (DOPC). These results indicate that the positively charged Skp targets OmpA to a negatively charged membrane, which facilitates the release of OmpA from its complex with Skp for subsequent folding and membrane insertion. The dual functionality of Skp as a chaperone and as a targeting protein is ideal to mediate the transport of OmpA and other outer membrane proteins across the periplasm in a folding-competent form to the outer membrane, which is negatively charged on its periplasmic side. OmpA ( $\mathrm{p} I$ 5.5) folded most efficiently above its isoelectric point. In the absence of Skp and in contrast to folding into DOPC bilayers, insertion and folding of OmpA were retarded for membranes containing DOPG at neutral or basic $\mathrm{pH}$ because of electrostatic repulsion. When folding of OmpA was performed near its isoelectric point, urea dilution led to a more compact aqueous form of OmpA previously characterized by fluorescence, which folded at a much slower rate. Under conditions where two different aqueous conformations of OmpA coexisted, e.g., in the titration region of OmpA, the last step of OmpA folding could be well described by two parallel pseudo-first-order kinetic phases. In this kinetic model, the contribution of the faster folding process, but not the changes in the rate constants, determined the folding yields obtained at different $\mathrm{pH}$. The faster phase dominated when the experimental conditions favored the less compact form of aqueous OmpA.
\end{abstract}

Insertion and folding of membrane proteins into membranes are not well understood but important for membrane biogenesis and cell growth. In Gram-negative bacteria, assembly of transmembrane proteins into the outer membrane $(\mathrm{OM})^{1}$ takes place posttranslationally after their translocation across the cytoplasmic membrane in unfolded form via the SecYEG translocon. Passage of outer membrane proteins (OMPs) through the periplasm is facilitated by periplasmic chaperones, which was demonstrated in genetic studies for the $17 \mathrm{kDa}$ protein $(\mathrm{Skp})(1,2)$ and the survival factor A (SurA) (3, 4). Deletion of skp or surA in Escherichia coli resulted in reduced concentrations of OMPs in the OM $(1,3-6)$. How these periplasmic molecular chaperones work in the absence of ATP is largely unknown.

\footnotetext{
†This work was supported by Grant KL1024/4-1/2 to J.H.K. from the Deutsche Forschungsgemeinschaft (DFG).

*Address correspondence to this author. Tel: +49-7531-882291. Fax +49-7531-883183. E-mail: joerg.helmut.kleinschmidt@uni-konstanz.de.

Abbreviations: CD, circular dichroism; DOPC, 1,2-dioleoyl-sn-glycero3-phosphocholine; DOPE, 1,2-dioleoyl-sn-glycero-3-phosphoethanolamine; DOPG, 1,2-dioleoyl-sn-glycero-3-phosphoglycerol; FomA, major outer membrane protein A of Fusobacterium nucleatum; LPS, lipopolysaccharide; OM, outer membrane; OMP, outer membrane protein; OmpA, outer membrane protein A of Escherichia coli; PE, phosphatidylethanolamine; PG, phosphatidylglycerol; SDS-PAGE, sodium dodecyl sulfatepolyacrylamide gel electrophoresis; Skp, the seventeen kDa protein; SUVs, small unilamellar vesicles; TMD, transmembrane domain.
}

OMPs can be unfolded in $8 \mathrm{M}$ urea solution. They fold spontaneously to their active forms upon urea dilution in the presence of detergent micelles (for a review see ref 7). Some OMPs, e.g., outer membrane protein A (OmpA) (8) or PagP (9) from E. coli, the major OMP from Fusobacterium nucleatum (FomA) (10), or the voltage-dependent anion-selective channel, isoform 1 of human mitochondria (11) also insert spontaneously into preformed lipid bilayers. This has facilitated detailed examinations of membrane protein folding by biophysical methods. However, insertion of OMPs into lipid bilayers is slow (12) and depends on the lipids and bilayer properties; see, e.g., refs 13 and 14. OmpA has emerged as an important model to examine the folding mechanism of $\beta$-barrel membrane proteins into lipid membranes $(12,13,15-17)$ (for a review, see ref 18). OmpA folding requires the hydrophobic core of either a detergent micelle or a lipid bilayer (17).

Upon urea dilution, Skp binds to unfolded OmpA, and soluble stable complexes are formed. This allowed investigations on the folding of OmpA into lipid membranes from its chaperonebound form, replacing urea by Skp for OmpA solubilization (19). To our knowledge, this is currently the only established minimal model system to investigate folding of a chaperone-bound membrane protein into lipid bilayers in detail. Only the OMP, Skp, and a preformed lipid bilayer are required, which greatly 
facilitates investigations of the folding mechanism by spectroscopic methods. Fluorescence spectroscopy indicated that Skp forms stable 3:1 complexes with OmpA $(19,20)$. Later, crystal structures (PDB entries 1sg2 and $1 \mathrm{u} 2 \mathrm{~m}$ ) revealed that Skp is a homotrimer (21-23). Skp-bound OmpA folded slower than ureaunfolded OmpA (19), which was unexpected since Skp is a periplasmic chaperone for OMPs, preserving them in a foldingcompetent conformation for membrane insertion. Interestingly, the simultaneous presence of Skp and lipopolysaccharide (LPS), a component of the OM, increased the folding rates and yields of OmpA beyond those obtained with urea-unfolded OmpA alone (19).

More recently, we showed that the Skp trimer forms 1:1 complexes with a range of OMPs from various bacteria at nanomolar affinity (20). However, OMPs were neither bound below $\mathrm{pH} 5.5$ nor above $\mathrm{pH}$ 10, i.e., under conditions where Skp $(\mathrm{p} I \sim 10.5)$ and $\mathrm{OMPs}(\mathrm{p} I<6(18,24))$ were no longer oppositely charged. Furthermore, at $\mathrm{pH} 7$, the affinity of Skp for OMPs decreased with increasing ionic strength in solution (20). In conclusion, Skp binding to OMPs was mediated largely by electrostatic interactions, although contributions of hydrophobic interactions to binding have not been ruled out.

In the present work, we examined the role of charge-charge interactions for the folding and insertion of Skp-bound OmpA. Our hypothesis was that the negative electrostatic surface potential of the periplasmic leaflet of the OM might attract $\mathrm{Skp}_{3} \cdot \mathrm{OMP}$ complexes, since Skp is a highly positively charged chaperone. For comparisons, folding was investigated for urea-unfolded and for Skp-bound OmpA over a broad $\mathrm{pH}$ range and with neutral and negatively charged lipid membranes. To examine Skp function and the basis for the chaperone-assisted folding of OMPs like OmpA, we also analyzed the folding kinetics of OmpA in the absence and in the presence of Skp or both Skp and LPS, since in our previous work LPS strongly modulated the properties of the $\mathrm{Skp}_{3} \cdot$ OmpA complex (20) and OmpA folding behavior (19).

\section{MATERIALS AND METHODS}

Kinetics of Omp A Folding Analyzed by SDS-Polyacrylamide Gel Electrophoresis. In our previous work (13), folding of OmpA was analyzed from the concentration dependence of OmpA folding into lipid membranes using the method of initial rates (25). Briefly, OmpA folding into membranes was described by the reaction:

$$
\mathrm{U}+\mathrm{L} \rightarrow \mathrm{F} \cdot \mathrm{L}
$$

where $\mathrm{U}$ is the unfolded OmpA, $\mathrm{L}$ the lipid of the membrane, and $\mathrm{F} \cdot \mathrm{L}$ the folded and membrane-inserted OmpA with bound lipid from the membrane. Folding rates of OmpA depended on the concentration of unfolded OmpA, [U], and on the concentration of the unbound lipid, [L], and therefore followed a second-order rate law:

$$
\frac{\mathrm{d}[\mathrm{F} \cdot \mathrm{L}]}{\mathrm{d} t}=-\frac{\mathrm{d}[\mathrm{U}]}{\mathrm{d} t}=k_{2 . \mathrm{ord}}[\mathrm{U}][\mathrm{L}]
$$

where $[\mathrm{F} \cdot \mathrm{L}]$ is the concentration of folded OmpA with bound lipid and $k_{2 . \text { ord }}$ the second order rate constant. At high lipid/OmpA ratios, [L] remained largely constant while OmpA folded. Therefore, the reaction was equally well described by a pseudo-first-order rate law:

$$
\frac{\mathrm{d}[\mathrm{F} \cdot \mathrm{L}]}{\mathrm{d} t}=-\frac{\mathrm{d}[\mathrm{U}]}{\mathrm{d} t}=k[\mathrm{U}]
$$

where $k=k_{2 . \text { ord }}[\mathrm{L}]$ is the pseudo-first-order rate constant. Integration of eq 2 leads to the single-exponential time course of folding of
OmpA:

$$
[\mathrm{U}](t)=[\mathrm{U}]_{0} \exp (-k t) \text { and }[\mathrm{F} \cdot \mathrm{L}]=U_{0}\{1-\exp (-k t)\}
$$

This single-exponential time course was previously confirmed for OmpA folding into lipid bilayers of short-chain phospholipids at basic $\mathrm{pH}(13)$.

Here, OmpA folding into the longer chain dioleoylphospholipids was monitored as a function of time by electrophoresis over a wide $\mathrm{pH}$ range. Native OmpA and refolded OmpA migrate at $30 \mathrm{kDa}$, if not heat-denatured prior to electrophoresis, whereas unfolded forms or aqueous intermediates of OmpA migrate at $35 \mathrm{kDa}$ (26). The $30 \mathrm{kDa}$ form has been shown by Raman, FT-IR, and $\mathrm{CD}$ spectroscopy $(8,17,27-30)$, by phage inactivation assays (26), and by single channel conductivity measurements (31) to strictly correspond to natively structured and functional OmpA. Under some experimental conditions chosen in this study, the observed folding kinetics of OmpA could not be satisfactorily fitted by single-exponential fit functions, corresponding to singlestep first-order kinetics. Instead, two kinetic phases of OmpA folding were necessary to describe the conversion of the $35 \mathrm{kDa}$ band to the folded form of OmpA migrating at $30 \mathrm{kDa}$. Since no other bands were detected on the gel and the $30 \mathrm{kDa}$ band strictly corresponds to stably refolded OmpA, these phases had to be parallel, arising from at least two different forms of OmpA that were not stable against SDS-PAGE and migrated both at $35 \mathrm{kDa}$. Depending on $\mathrm{pH}$, two aqueous forms of OmpA have been observed previously, characterized by different fluorescence properties. These forms titrated between $\mathrm{pH} 4$ and $\mathrm{pH} 7$ as shown by Qu et al. (20). SDS-PAGE monitors only the last folding stage on a minute time scale $(12,13)$. Therefore, the two parallel phases, observed here for the last folding stage of OmpA, arise from two distinct aqueous folding intermediates, $\mathrm{IM}_{1}$ and $\mathrm{IM}_{2}$, which are both converted to folded OmpA but at different rates:

$$
\begin{array}{cc}
\mathrm{IM}_{1} \stackrel{k_{\mathrm{f}}}{\rightarrow}(\mathrm{F} \cdot \mathrm{L})_{\mathrm{f}} & \text { (fast) } \\
\mathrm{IM}_{2} \stackrel{k_{\mathrm{s}}}{\rightarrow}(\mathrm{F} \cdot \mathrm{L})_{\mathrm{s}} & \text { (slow) }
\end{array}
$$

$(\mathrm{F} \cdot \mathrm{L})_{\mathrm{f}}$ and $(\mathrm{F} \cdot \mathrm{L})_{\mathrm{s}}$ both describe folded OmpA, $[\mathrm{F} \cdot \mathrm{L}](t)=$ $\left[(\mathrm{F} \cdot \mathrm{L})_{\mathrm{f}}\right](t)+\left[(\mathrm{F} \cdot \mathrm{L})_{\mathrm{s}}\right](t)$, formed in the faster (index $\mathrm{f}$ ) and in the slower (index s) parallel phase. In the electrophoretic analysis, the sum $\left[(\mathrm{IM})_{1}\right](t)+\left[(\mathrm{IM})_{2}\right](t)+[\mathrm{F} \cdot \mathrm{L}](t)$ is equal to the total concentration of OmpA in the system. The rate of folding into the $30 \mathrm{kDa}$ form is given by the sum of two independent pseudo-firstorder folding phases with the rate constants $k_{\mathrm{f}}$ for the fast and $k_{\mathrm{S}}$ for the slow folding step:

$$
\begin{aligned}
\frac{\mathrm{d}[\mathrm{F} \cdot \mathrm{L}]}{\mathrm{d} t}=\frac{\mathrm{d}\left[(\mathrm{F} \cdot \mathrm{L})_{1}\right]}{\mathrm{d} t} & +\frac{\mathrm{d}\left[(\mathrm{F} \cdot \mathrm{L})_{2}\right]}{\mathrm{d} t}=-\frac{\mathrm{d}\left[\mathrm{IM}_{1}\right]}{\mathrm{d} t}-\frac{\mathrm{d}\left[\mathrm{IM}_{2}\right]}{\mathrm{d} t} \\
& =k_{\mathrm{f}}\left[\mathrm{IM}_{1}\right]+k_{\mathrm{S}}\left[\mathrm{IM}_{2}\right]
\end{aligned}
$$

Integration and substitution of the concentrations by mole fractions leads to

$$
X_{\mathrm{F} . \mathrm{L}}=1-\left[X_{\mathrm{IM}_{1}, 0} \exp \left(-k_{\mathrm{f}} t\right)+X_{\mathrm{IM}_{2}, 0} \exp \left(-k_{\mathrm{s}} t\right)\right]
$$

where $X_{\mathrm{IM}_{1}, 0}$ and $X_{\mathrm{IM}_{2}, 0}$ are the extrapolated mole fractions of the intermediate forms $\mathrm{IM}_{1}$ and $\mathrm{IM}_{2}$ for $t \rightarrow 0 \mathrm{~min}$. Since at $t=0 \mathrm{~min}$ the fraction of folded OmpA is $X_{\mathrm{F} \cdot \mathrm{L}, 0}=0$, it follows that the sum $X_{\mathrm{IM}_{1}, 0}+X_{\mathrm{IM}_{2}, 0}=1$ and the equation can be further simplified to

$$
X_{\mathrm{F} . \mathrm{L}}(t)=1-\left[A_{\mathrm{f}} \exp \left(-k_{\mathrm{f}} t\right)+\left(1-A_{\mathrm{f}}\right) \exp \left(-k_{\mathrm{s}} t\right)\right]
$$

where $A_{\mathrm{f}}$ is the contribution of the faster phase in the last folding stage to the overall folding yield of OmpA. This model also 
includes the possibility that not all OmpA folds, i.e., $k_{\mathrm{s}}=0$. Equation 6 can then be transformed to

$$
X_{\mathrm{FP}}(t)=A_{\mathrm{f}}\left[1-\exp \left(-k_{\mathrm{f}} t\right)\right]
$$

In this case, $A_{\mathrm{f}}$ corresponds to the final yield of folded OmpA. All curve fits were performed using IGOR Pro 6.0 (Wavemetrics).

Purification of Skp, OmpA, and R-LPS. Skp was isolated from $E$. coli and purified as described previously (19). OmpA was overexpressed and purified in denatured form in $8 \mathrm{M}$ urea as reported earlier (8). The concentrations of Skp and OmpA were determined using the method of Lowry et al. (32). E. coli rough mutant F576 was cultivated as described previously (33), and its LPS (R2 core type, $M_{\mathrm{r}} \sim 3900 \mathrm{~g} / \mathrm{mol}$ ) was isolated as reported (34).

Preparation of Lipid Vesicles. 1,2-Dioleoyl-sn-glycero3-phosphocholine (DOPC), 1,2-dioleoyl-sn-glycero-3-phosphoethanolamine (DOPE), and 1,2-dioleoyl-sn-glycero-3-phosphoglycerol (DOPG) were purchased from Avanti Polar Lipids (Alabaster, AL). Small unilamellar vesicles (SUVs) in $10 \mathrm{mM}$ buffer containing 2 mM EDTA were prepared as described (19). The buffer was sodium citrate at $\mathrm{pH}$ 5.5-6.5, HEPES at $\mathrm{pH} 7$, Tris at $\mathrm{pH} 8$, or sodium borate at $\mathrm{pH} 9$ and 10 .

Kinetics of Tertiary Structure Formation by Electrophoresis. To compare folding kinetics at a selected $\mathrm{pH}$ in the absence and in the presence of either Skp or both Skp and LPS, folding experiments were performed in parallel with lipid bilayers (SUVs) from the same preparation (19). Insertion and folding of urea-unfolded OmpA into SUVs were initiated by rapidly mixing OmpA with an excess volume of lipid bilayers in buffer for strong urea dilution. Folding of OmpA from the complex with Skp was performed by first adding the unfolded OmpA to urea-free buffer containing Skp, reducing the urea concentration 12-fold. SUVs were then added to initiate folding and insertion. Alternatively, OmpA.Skp complexes were reacted with a solution of LPS followed by immediate addition of SUVs. The final concentrations in the folding reactions were $7.1 \mu \mathrm{M}$ OmpA, $1.4 \mathrm{mM}$ lipid, and, where present, $28.4 \mu \mathrm{M} \mathrm{Skp}$ and $35.5 \mu \mathrm{M} \mathrm{LPS}$, corresponding to molar ratios of 1 OmpA, 200 lipids, 4 Skp, and 5 LPS. The final urea concentration was $0.5 \mathrm{M}$ in all experiments. Folding kinetics was monitored over $240 \mathrm{~min}$ after bilayer addition at $30^{\circ} \mathrm{C}$ and analyzed by electrophoresis as described previously $(10,12,13,35)$ (for a review, see ref 18). Sodium dodecyl sulfate-polyacrylamide gel electrophoresis (SDS-PAGE) $(36,37)$ was performed without heat denaturation of the samples.

\section{RESULTS}

The Folding Kinetics of OmpA into Lipid Bilayers Is Strongly $p H$ Dependent. For folding experiments, OmpA was isolated in unfolded form in $8 \mathrm{M}$ urea. Previous experiments have shown that after urea dilution in aqueous solution the tryptophan fluorescence maximum of OmpA was located at 336-338 nm when measured below pH 5.5 (20). This indicated a compact, hydrophobic environment of the transmembrane $\beta$-strand regions. In contrast, when the same experiment was performed at $\mathrm{pH}>6$, fluorescence intensities were lower and the emission maxima were at $343-344 \mathrm{~nm}$, indicating partial tryptophan exposure to water in a less compact form, likely arising from intramolecular repulsions between predominantly negatively charged side chains. The fluorescence data by Qu et al. (20) indicated that these two major conformers titrate between $\mathrm{pH} 4$ and $\mathrm{pH} 7$, which includes the calculated $\mathrm{p} I$ of OmpA (20).
To examine differences in folding of these OmpA conformations into lipid membranes, we determined folding kinetics of OmpA as a function of $\mathrm{pH}$. We used lipid bilayers composed of dioleoylphosphatidylcholine (DOPC), dioleoylphosphatidylethanolamine (DOPE), and dioleoylphosphatidylglycerol (DOPG) at molar ratios of 5:3:2, since the periplasmic leaflet of the E. coli OM has a negative electrostatic surface potential, containing $\sim 20 \%$ negatively charged phosphatidylglycerol (PG) and diphosphatidylglycerol (cardiolipin) in addition to phosphatidylethanolamine (PE). Folding kinetics of OmpA was analyzed by SDS-PAGE on samples that were not heated prior to electrophoresis, taking advantage of the different electrophoretic mobilities of folded $(30 \mathrm{kDa})$ and unfolded OmpA (35 kDa) as described, e.g., in refs 13,19 , and 35 (for a review, see ref 18). Differences in migration of folded and unfolded forms are common for OMPs and were reported for OmpG (38), FomA (39), PagP (9), and others.

Figure 1 shows the kinetics of OmpA folding into DOPC/ DOPE/DOPG bilayers of small unilamellar vesicles (SUVs) at $\sim 200$ lipids/OmpA over $240 \mathrm{~min}$ after urea dilution. Time courses were monitored at various $\mathrm{pH}$ between 5 and 10 and analyzed by SDS-PAGE (Figure 1A) and densitometry (Figure 1B,C). Lower yields of folded OmpA and reduced folding rates were obtained for the more compact form of OmpA at $\mathrm{pH} \leq 5.5$ close to $I_{\text {OmpA A }}$ At $\mathrm{pH} 5.5, \mathrm{OmpA}$ folded to less than $30 \%$ (Figure 1A, B). At $\mathrm{pH} 5$, folding was almost negligible, but this form still folded to more than $20 \%$ within 240 min into bilayers of shortchain lipids (not shown), for which faster kinetics were also reported at $\mathrm{pH} 10$ (13). In contrast, fast folding kinetics and yields of $\sim 90 \%$ folded OmpA were obtained when OmpA was negatively charged at $\mathrm{pH}$ 6.5. At neutral and at basic $\mathrm{pH}$, yields still exceeded $60 \%$. The large conformational differences between two major aqueous forms of OmpA observed by Qu et al. in fluorescence experiments (20) explain this $\mathrm{pH}$ dependence of the folding kinetics of OmpA. The folding rate of the more compact form was slower, likely because a larger number of conformational changes to invert the "outside-in" conformation of the transmembrane strands is needed for this form in comparison to the less compact form. The conformational state of OmpA in aqueous solution is therefore a major determinant of the rate of folding and insertion into membranes. In the $\mathrm{pH}$ region, where the two previously observed forms of OmpA coexist, they fold in parallel.

Skp Facilitates Folding of OmpA at Neutral or Basic $p H$. In previous studies at $\mathrm{pH} 7$, folding of OmpA into bilayers of DOPC/DOPE/DOPG (5:3:2) was retarded by Skp in the absence of negatively charged LPS but facilitated when Skp and LPS were both present (19).

To explore whether the net charge of the $\mathrm{Skp}_{3} \cdot \mathrm{OmpA}$ complex is important for the retarding effect of Skp in the absence of LPS, we next examined folding of Skp-bound OmpA at various $\mathrm{pH}$ (Figure 2). Folding of OmpA was initiated by the addition of preformed $\mathrm{Skp}_{3} \cdot \mathrm{OmpA}$ complexes to bilayers of DOPC/DOPE/ DOPG (5:3:2) (SUVs). Compared to urea-unfolded OmpA, which folded to $\sim 90 \%$ at $\mathrm{pH} 6.5$ (Figure 1), folding of Skpbound OmpA was strongly retarded below $\mathrm{pH} 7$, and folding yields never exceeded 20\% (see Figure 2A and the gels in Figure S1B of the Supporting Information). At $\mathrm{pH} 7$ and above (Figures 2B and S1B), yields and rates were comparable to those of urea-denatured OmpA (Figure 1C). It is interesting that the region below $\mathrm{pH}$ 7, i.e., where folding was retarded, overlaps with the $\mathrm{pH}$ range, in which OmpA titrated between a more open and a more compact form, as observed by fluorescence in aqueous solution (20). In the previous study by Qu et al. (20), OmpA 


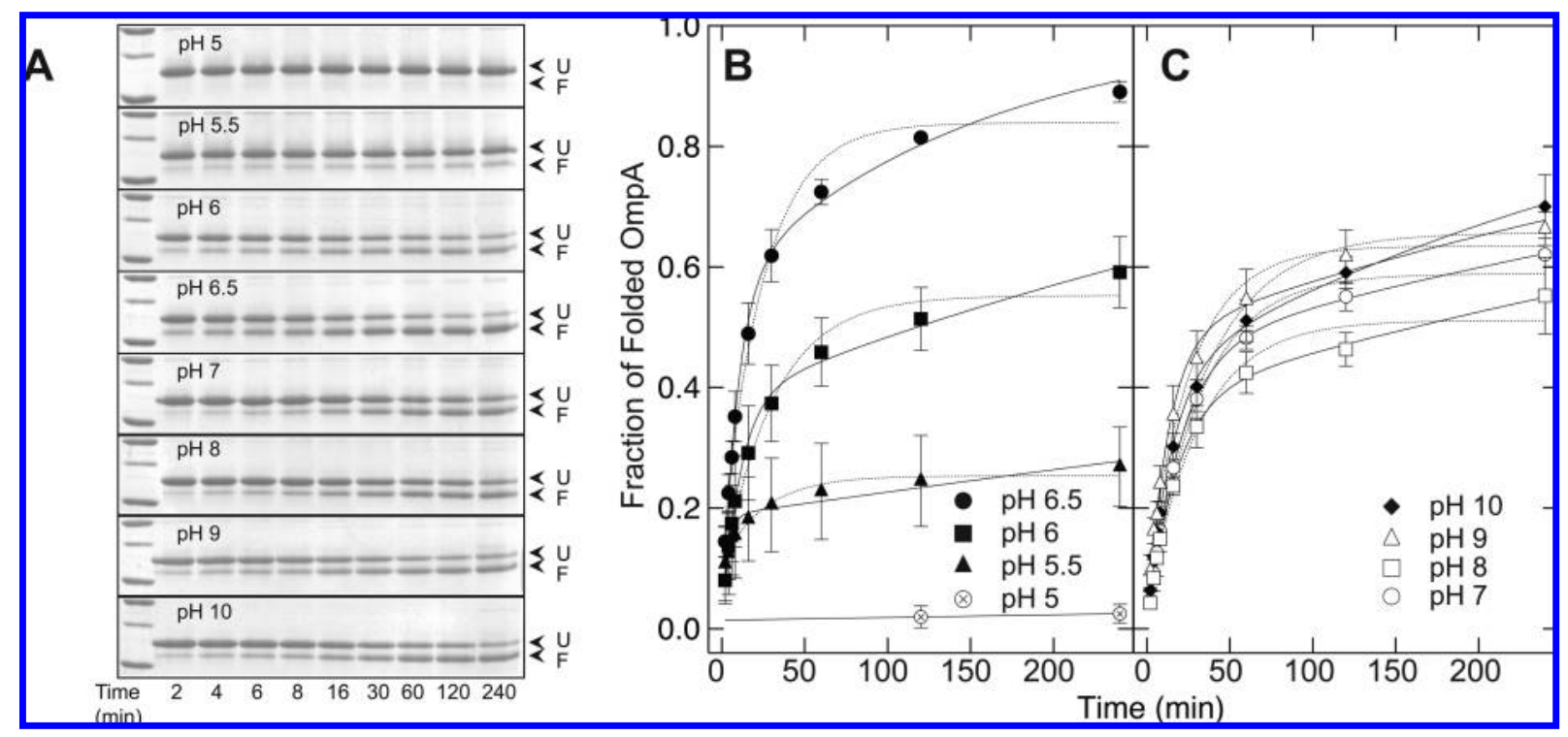

FIGURE 1: The kinetics of membrane protein folding of OmpA depends on $\mathrm{pH}$. (A) SDS-PAGE analysis of the time courses of OmpA (7.1 $\mu \mathrm{M}$ ) folding into lipid bilayers (1.4 mM lipid) of DOPC/DOPE/DOPG at a molar ratio of 5:3:2 at $30{ }^{\circ} \mathrm{C}$ and at the indicated pH. Folding was monitored from 2 to 240 min after mixing urea-unfolded OmpA with preformed lipid bilayers as described in Materials and Methods. The times are indicated at the bottom for each lane. In each gel, the first lane contained the protein markers. Unfolded OmpA (U) migrated at $35 \mathrm{kDa}$ and folded OmpA (F) at $30 \mathrm{kDa}(19)$. (B, C) The fraction of folded OmpA was analyzed by densitometry of the gels shown in (A) and plotted as a

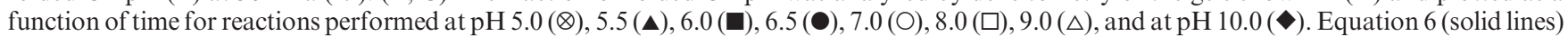
and single-exponential functions (dotted lines) were fitted to the experimental data using a nonlinear least-squares algorithm.

$\left(\mathrm{p} I_{\mathrm{OmpA}} \sim 5.5\right)$ and $\operatorname{Skp}\left(\mathrm{p} I_{\text {Skp }} \sim 10.5\right)$ formed stable complexes from $\mathrm{pH} \sim 5-6$ to $\mathrm{pH} \sim 10$. At $\mathrm{pH} 7$, the determined binding affinity was high with a dissociation constant $K_{\mathrm{D}}$ of $\sim 20 \mathrm{nM}$, indicating $98-99 \%$ of the OmpA is bound to Skp (20) according to the mass action law. This raised the question why OmpA insertion and folding into the membrane in the presence of Skp were favored at $\mathrm{pH} 7$ and above, but not below.

A Small LPS/OmpA Ratio Promotes Folding of OmpA from the Complex with Skp from $\mathrm{pH} 6$ to $\mathrm{pH} 8$. Previously, LPS from the OM of $E$. coli facilitated folding of OmpA into lipid bilayers from a complex with Skp at $\mathrm{pH} 7$ in particular at small ratios of 2-7 LPS/OmpA (19). We therefore investigated whether this effect of LPS on folding of Skp-bound OmpA is $\mathrm{pH}$ dependent. In comparison to folding of OmpA from the Skp complex in the absence of LPS (Figure 2A,B) and at a $\mathrm{pH}$ between 6 and 8, Skp-bound OmpA folded to higher yields and with faster rates into the same lipid bilayers when 5 LPS per OmpA were additionally present under otherwise identical conditions (Figure 2C,D and gels in Figure S1C). At pH 6.5, the yields were $74 \%$ in the presence but less than $20 \%$ in the absence of LPS, while at $\mathrm{pH} 7$, yields reached $86 \%$ in the presence but only $60 \%$ in the absence of LPS. Slightly smaller differences in rates and yields were also observed at $\mathrm{pH} 8$, with $\sim 79 \%$ yield of folded OmpA in the presence and $\sim 67 \%$ in the absence of LPS (Figure 2). At pH 9-10, Skp-bound OmpA folded to $\sim 70 \%$, and the changes caused by the additional presence of LPS were comparably small. The experimentally observed time courses of OmpA folding demonstrated that the negatively charged LPS increases the folding rates and yields of Skp-bound OmpA into bilayers composed of DOPC/DOPE/DOPG from $\mathrm{pH} 6$ to $\mathrm{pH} 9$ but not at $\mathrm{pH} 5$, i.e., close to $\mathrm{I}_{\mathrm{OmpA}}$, or at $\mathrm{pH} 10$, i.e., close to $\mathrm{p} I_{\text {Skp }}$. The strongly retarded folding of OmpA by Skp at $\mathrm{pH}$ 6-6.5 was no longer observed when LPS was present, which is consistent with recent observations that the addition of LPS changes the topology of the Skp.OmpA complex, partially exposing OmpA to the aqueous environment $(20,40)$.
Our results established a simple experimental protocol to study OmpA folding, in which urea is no longer required for OmpA solubilization and is substituted by Skp. This experimental design allows detailed studies on OMP folding involving only selected naturally occurring components, namely, the chaperone Skp, which replaces the chaotropic denaturant urea to keep OmpA soluble, and lipid membranes, which are necessary for folding of OmpA.

Folding of OmpA Shows a Different $p H$ Dependency for Neutral and for Charged Lipid Bilayers. To examine whether the negative electrostatic potential of the DOPC/DOPE/DOPG membranes is important for OmpA folding, experiments were repeated with DOPC bilayers, which do not carry a net charge. First, urea-unfolded OmpA was folded into DOPC membranes, and the fraction of folded OmpA was determined as a function of time (Figure 3A,B and the corresponding original gels in Figure $\mathrm{S} 2 \mathrm{~A}$ ). Folding yields after $240 \mathrm{~min}$ were high from $\mathrm{pH} 6$ to $\mathrm{pH} 10$, but at $\mathrm{pH}<6$, rates and yields were decreased (Figure 3A). At pH 7 and above (Figure 3B), yields of folded OmpA in DOPC membranes exceeded $90 \%$ and were consistently higher than folding yields observed for OmpA in the negatively charged DOPC/DOPE/DOPG bilayers (Figure 1C). In this case, the difference was not caused by the presence of DOPE, since the yields were high at $\mathrm{pH} 6.5$ for both DOPC and DOPC/DOPE/ DOPG bilayers and because similarly reduced yields were also observed for bilayers of DOPC and DOPG at a ratio of 7:3 (Figure 4). The reduced yields of folded OmpA in DOPC/DOPE/ DOPG or DOPC/DOPG bilayers vs pure DOPC bilayers are likely caused by electrostatic repulsion between negatively charged OmpA and the negatively charged lipid bilayers. For OmpA folding into DOPC/DOPE/DOPG bilayers, this repulsion was less pronounced at $\mathrm{pH} 6.5$ (Figure 1B). Upon lowering the $\mathrm{pH}$ from 8 to 5 , the five OmpA histidine side chains will protonate $(41,42)$, reducing the net negative charge of OmpA and the electrostatic repulsion by the membrane.

Skp Strongly Retards Folding of OmpA into Phosphatidylcholine Bilayers. To examine the role of the highly basic, 


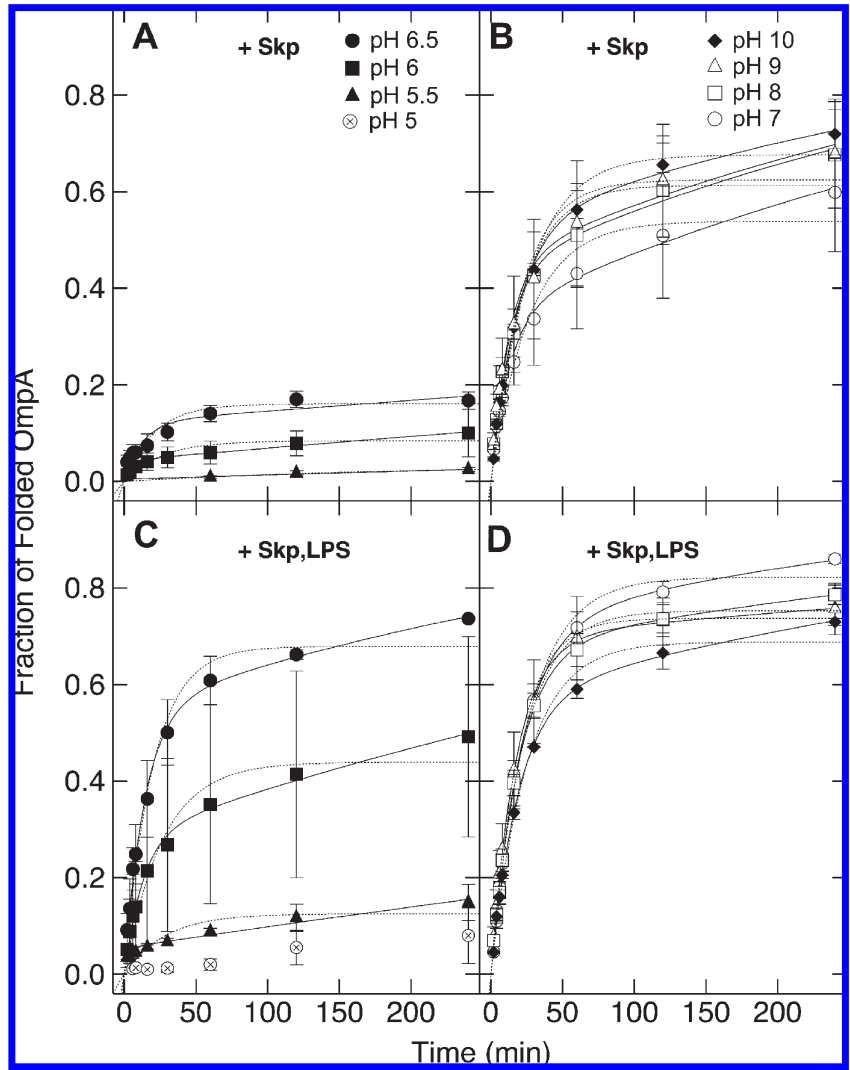

FIGURE 2: OmpA folding from a complex with $\mathrm{Skp}_{3}$ into negatively charged bilayers is retarded when LPS is absent but facilitated when LPS is present. Folding was monitored as a function of time after lipid addition over $240 \mathrm{~min}$ as described in the legend to Figure 1 and in the Materials and Methods section. Folding reactions were performed at pH $5(\otimes), 5.5(\mathbf{\Delta}), 6.0(\square), 6.5(\bullet), 7.0(\bigcirc), 8.0(\square), 9.0(\triangle)$, and 10.0 $(\checkmark)$. Representative gels are shown in Figure S1B,C of the Supporting Information. (A, B) Urea-unfolded OmpA was first reacted with a 4fold molar excess of Skp, diluting the urea 12 times. Lipid bilayers composed of DOPC/DOPE/DOPG $(5: 3: 2)$ were then added to initiate folding in the absence of LPS. (C, D) As in (A) and (B) but $\mathrm{Skp}_{3} \cdot$ OmpA complexes were reacted with $5 \mathrm{LPS} / \mathrm{OmpA}$ before lipid was added. Equation 6 (solid lines) or single-exponential functions (dotted lines) were fitted to the experimental data.

i.e., positively charged, periplasmic chaperone Skp for the folding of OmpA into lipid membranes in more detail, we repeated our folding experiments with Skp-bound OmpA (Figure 2A,B) but with neutral bilayers instead of charged DOPC/DOPE/DOPG membranes. Figure 3C,D and the corresponding gels shown in Figure S2B of the Supporting Information show the time courses of folding of Skp-bound OmpA into DOPC bilayers at different $\mathrm{pH}$. A striking difference in the folding kinetics of OmpA was that the initial steep increase in folding yields of OmpA previously observed for the charged DOPC/DOPE/DOPG bilayers (Figure 2B,D) was no longer observed for the neutral DOPC bilayers. A comparison between the two sets of experiments indicates that folding of Skp-bound OmpA into DOPC bilayers is much slower. Slow folding was observed over the entire $\mathrm{pH}$ range, although slightly faster rates were obtained at $\mathrm{pH} 6.5$ and at $\mathrm{pH} 10$.

In comparison to urea-unfolded OmpA (Figure 3A,B), folding of Skp-bound OmpA into DOPC bilayers was strongly retarded independent of $\mathrm{pH}$, and yields of folded OmpA were reduced, never exceeding $50 \%$ over the entire $\mathrm{pH}$ range even after $240 \mathrm{~min}$. The comparison further showed that the retarding effect of Skp was strongest between $\mathrm{pH} 6$ and $\mathrm{pH}$ 10, i.e., in between $\mathrm{p} I_{\mathrm{OmpA}}$

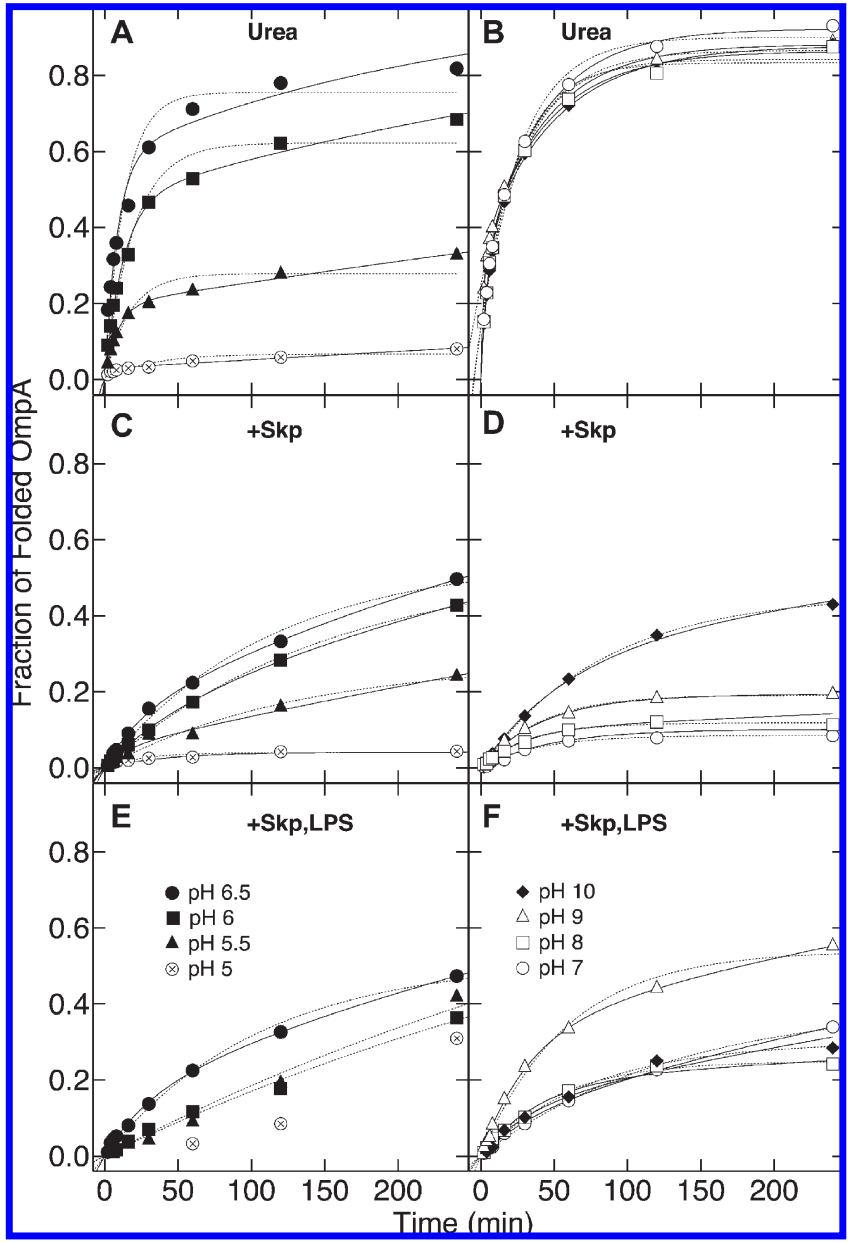

FIgURE 3: The kinetics of OmpA folding into lipid bilayers of DOPC is strongly retarded by Skp even when LPS is present. (A, B) Folding of OmpA from urea. (C, D) Folding of OmpA from the $\mathrm{Skp}_{3} \cdot \mathrm{OmpA}$ complex in the absence of LPS. (E, F) Folding from the $\mathrm{Skp}_{3} \cdot \mathrm{OmpA}$ complex in the presence of LPS. Kinetics were obtained as described for Figures 1 and 2 at acidic (A, C, and E) and at neutral or basic $\mathrm{pH}$ $(\mathrm{B}, \mathrm{D}$, and F), namely, at pH $5(\otimes), 5.5(\mathbf{\Delta}), 6.0(\mathbf{\square}), 6.5(\bullet), 7.0(\mathrm{O})$, $8.0(\square), 9.0(\triangle)$, and $10.0(\diamond)$. The corresponding SDS-polyacrylamide gels are shown in Figure S2 of the Supporting Information. Equation 6 (solid lines) or single-exponential functions (dotted lines) were fitted to the experimental folding kinetics.

and $\mathrm{pI}_{\mathrm{Skp}}$, the $\mathrm{pH}$ range where Skp and OmpA are oppositely charged.

A Small LPS/OmpA Ratio Is Insufficient to Fully Compensate the Retarding Effect of Skp on OmpA Folding into Neutral DOPC Bilayers. When Skp-bound OmpA was folded into DOPC/DOPE/DOPG membranes, we observed that the presence of a 5 -fold molar excess of LPS per OmpA promoted folding of OmpA between $\mathrm{pH} 6$ and pH 9. To examine whether this effect is independent of the lipid bilayer used for insertion and folding of Skp-bound OmpA, we repeated this experiment but with pure DOPC bilayers (Figure 3E,F and Figure S2C of the Supporting Information). In the acidic $\mathrm{pH}$ range, LPS stimulated folding of Skp-bound OmpA into DOPC bilayers at $\mathrm{pH} 5$ (from $4 \%$ to $31 \%$, i.e., $\sim 8$-fold) and slightly at $\mathrm{pH} 5.5$ (from $24 \%$ to $42 \%$ ) but not at $\mathrm{pH} 6$ and 6.5 (Figure 3E). LPS also improved folding yields at $\mathrm{pH} 7$ (from $8.5 \%$ to $34 \%$, i.e., $\sim 4$-fold), at $\mathrm{pH} 8$ (from $11 \%$ to $24 \%$ ), and at $\mathrm{pH} 9$ (from $19 \%$ to $55 \%$ ) but not at $\mathrm{pH}$ 10. However, on an absolute scale and in comparison to folding of urea-unfolded OmpA, Skp retarded folding into the neutral DOPC bilayers from $\mathrm{pH} 6$ to $\mathrm{pH} 10$, whether small 


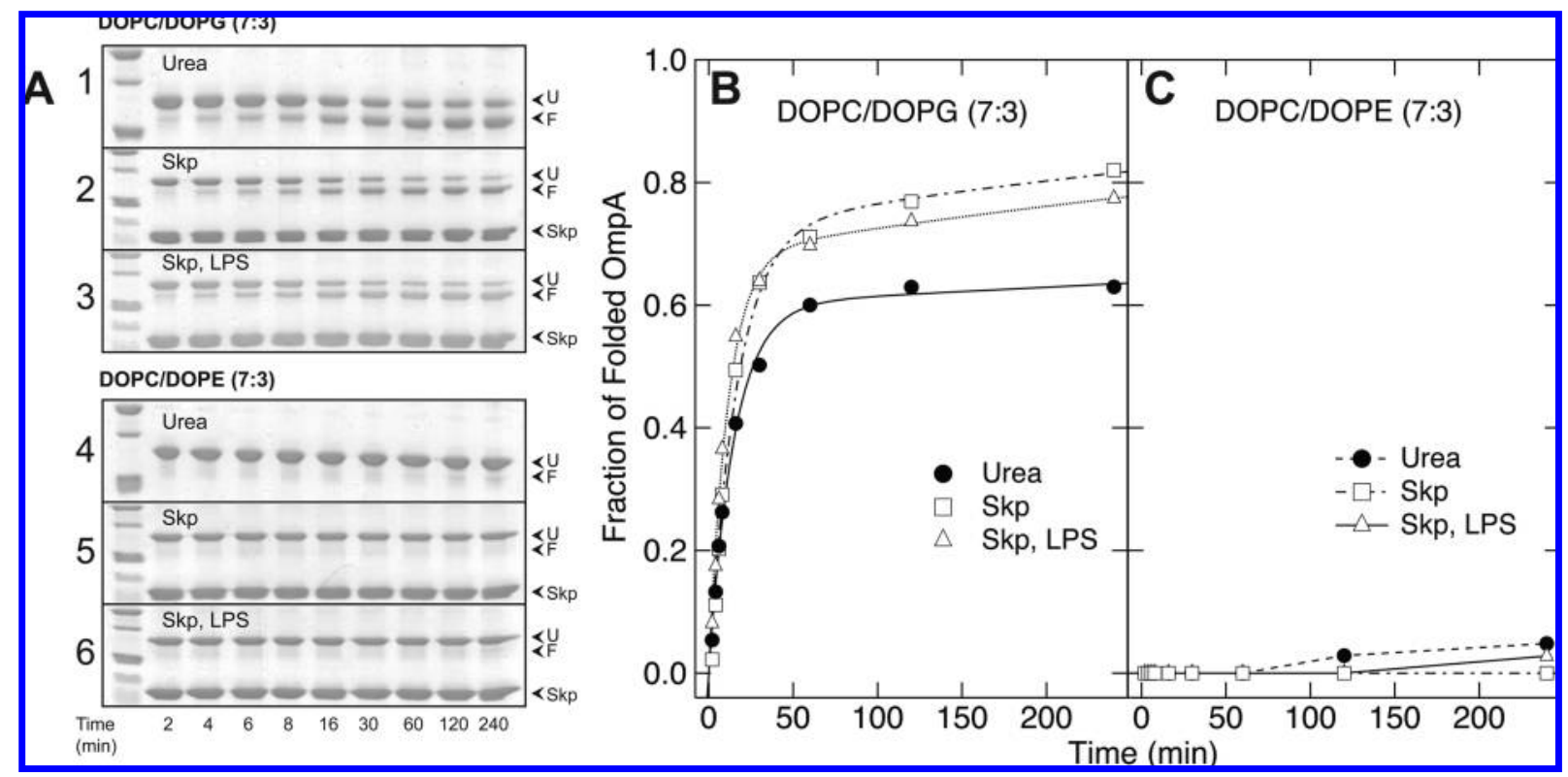

FIgURE 4: The periplasmic chaperone Skp and LPS facilitate OmpA folding into bilayers composed of DOPC/DOPG (7:3) but inhibit folding into bilayers composed of DOPC/DOPE (7:3). (A) SDS-PAGE analysis of the time courses of OmpA folding into DOPC/DOPG bilayers (gels $1-3)$ and into DOPC/DOPE bilayers (gels 4-6) at a molar L/P ratio of $200 . \mathrm{OmpA}(7.1 \mu \mathrm{M})$ and lipid $(1.4 \mathrm{mM})$ were reacted at $30^{\circ} \mathrm{C}$ and at $\mathrm{pH}$ 8.0. Reactions were performed in the absence and in the presence of Skp and in the presence of both Skp and LPS as described in the legends for Figures 1-3. The fraction of folded OmpA at various times after addition of lipid bilayers was analyzed by densitometry of the gels shown in (A) to obtain folding kinetics of OmpA. (B) Folding kinetics of OmpA into charged DOPC/DOPG bilayers. (C) Folding kinetics of OmpA into neutral DOPC/DOPE bilayers. Folding of OmpA into lipid bilayers was analyzed for urea-unfolded OmpA $(\bullet)$ and for OmpA bound in $\mathrm{Skp}_{3} \cdot \mathrm{OmpA}$ complexes either in the absence ( $\square$ ) or in the presence of LPS $(\triangle)$.

amounts of LPS were present or not. In comparison to ureaunfolded OmpA in the absence of Skp and LPS, the presence of both Skp and LPS led to slightly higher yields and faster rates only at $\mathrm{pH} \sim 5-5.5$.

Folding of Skp-Bound OmpA into Lipid Bilayers IS Facilitated by $P G$ but Inhibited by $P E$. The strongly reduced rates and yields of folding of Skp-bound OmpA into DOPC membranes (Figure 3D) at neutral or basic $\mathrm{pH}$ were in stark contrast to those observed for OmpA folding from the Skp complex into DOPC/DOPE/DOPG membranes (Figure 2B). Control experiments with lipid bilayers composed of binary mixtures of either DOPC and DOPG (7:3) (Figure 4A,B) or DOPC and DOPE (7:3) (Figure 4A and C) confirmed that the increased folding yields observed for Skp-bound OmpA into DOPC/DOPE/DOPG membranes were caused by the presence of negatively charged DOPG and not by the presence of DOPE. At $\mathrm{pH}$ 8, the presence of DOPG facilitated folding of Skp-bound OmpA (Figure 4). The Skp-bound OmpA also folded faster than urea-unfolded OmpA (Figure 4A,B). In contrast, folding of OmpA was most strongly inhibited when lipid membranes were composed of binary mixtures of DOPC and DOPE (7:3) (Figure 4A,C), regardless of whether urea-unfolded OmpA or Skp-bound OmpA was used in folding experiments. The comparison of Figure 4C with Figure 3B indicates that DOPE is a very effective inhibitor for OmpA insertion and folding into lipid bilayers composed of DOPC and DOPE alone.

Positively Charged Skp and Electrostatic Interactions with Negatively Charged Membranes Promote Folding of $O m p A$. The comparison of all experiments presented in this study shows that folding of Skp-bound OmpA into lipid membranes is greatly facilitated when the membrane contains the negatively charged DOPG. In this case OmpA folded to high yields, $\sim 60-90 \%$ at $\mathrm{pH} \geq 7.0$ in the absence (Figure $2 \mathrm{~B}$ ) and at $\mathrm{pH} \geq 6.5$ in the presence (Figure 2C,D) of negatively charged
LPS, i.e., in the $\mathrm{pH}$ region where the less compact/negatively charged form of OmpA dominates. Skp-bound OmpA also folded at $\mathrm{pH} \leq 5.5$ into neutral DOPC bilayers when LPS was also present, but the yields were generally smaller than $\sim 42 \%$ and folding was slow under this condition (Figures $2 \mathrm{C}$ and $3 \mathrm{E}$ ). The differences in the OmpA folding yields with negatively charged compared to neutral lipid bilayers indicate that electrostatic attraction or interactions between the $\mathrm{Skp}_{3} \cdot \mathrm{OmpA}$ complex and the surface of a negatively charged lipid bilayer strongly improve OmpA folding rates and yields at or above $\mathrm{pH} 6.5$. OmpA still folded into neutral lipid bilayers but at much slower rates and to much lower yields: At $\mathrm{pH} 7$ and in the absence of LPS, Skp-bound OmpA folded to $8.4 \%$ into pure DOPC bilayers (Figure 3D), while yields were $60 \%$, i.e., $~ 7$-fold higher, in charged DOPC/DOPE/DOPG membranes (Figure 2B) under otherwise identical conditions.

\section{DISCUSSION}

In our present detailed study we have examined the biochemical and biophysical basis of folding of OmpA from a complex with the chaperone Skp. Our observations are a major advancement in understanding insertion and folding of OMPs: (1) Different conformational forms of aqueous OmpA, observed between $\mathrm{pH} 5$ and $\mathrm{pH} 10$, fold and insert into membranes with different rates. This is not only relevant for folding into lipid membranes, but also relevant for folding in vivo since $E$. coli can grow between $\mathrm{pH} 4.4$ and $\mathrm{pH} 9.2(43)$ and the $\mathrm{pH}$ in the periplasm corresponds to the $\mathrm{pH}$ outside the cell (44). (2) The folding kinetics of OmpA into DOPC/DOPE/DOPG bilayers, observed by electrophoresis, is described by parallel folding processes, in particular in the titration region around $\mathrm{p} I_{\mathrm{OmpA}}$. (3) The faster folding process is favored when the solubility of OmpA is increased at basic $\mathrm{pH}$, i.e., when OmpA is negatively charged or when OmpA is solubilized by 
complex formation with Skp. (4) Unlike urea-unfolded OmpA, $\mathrm{Skp}_{3} \cdot$ OmpA complexes strongly favor interactions with negatively charged membranes in comparison to neutral membranes. This function of Skp, to deliver OMPs to charged membranes, appears to be as important for membrane protein folding of OmpA as its function to keep OMPs soluble. (5) PE completely inhibits folding of Skp-bound OmpA.

Folding of OmpA Displays a Complex pH Dependence. OmpA titrates between a more compact form at $\mathrm{pH} 4-5$ and a less compact form at $\mathrm{pH} \geq 6$ (20). Therefore, urea-unfolded OmpA folded faster at $\mathrm{pH} \geq 6$ (Figures $3 \mathrm{~A}, \mathrm{~B}$ and 1 ). The folding kinetics of the Skp-bound form of OmpA was also faster at $\mathrm{pH} \geq 7$ (Figure 2), indicating that Skp promoted folding of the less compact, unfolded form of OmpA into dioleoylphospholipid membranes at $\mathrm{pH} 7$ or above but not below. The binding affinity of Skp for OmpA is in the nanomolar range at $\mathrm{pH} 7$ (20). However, the retarded folding at $\mathrm{pH} \sim 6-6.5$, a region where histidine protonation or deprotonation alters the charge state of OmpA, suggests that binding of Skp to OmpA is strongest under mildly acidic conditions, possibly involving histidines of the TMD of OmpA in binding.

The $\mathrm{pH}$ dependence of the OmpA folding kinetics is interesting, because cells like $E$. coli (which contains PG in its $\mathrm{OM}$ ) can grow at $\mathrm{pH}$ between $\sim 4.4$ and $\sim 9.2(43)$. The $\mathrm{pH}$ in the periplasm corresponds to the $\mathrm{pH}$ outside the cells, while $\mathrm{pH}$ homeostasis is much better controlled in the cytosol (44). The expression levels of OMPs as a function of the external $\mathrm{pH}$ depend on the OMP, and elevated levels of OmpA in E. coli were reported at $\mathrm{pH} \geq 7$ (43). This is consistent with our present observations that insertion and folding of Skp-bound OmpA into negatively charged bilayers (Figures 2 and 4), containing PG in a similar concentration like the $\mathrm{OM}$ of $E$. coli, are favored at neutral to basic $\mathrm{pH}$. E. coli can also grow at a $\mathrm{pH}<6$. Therefore, other periplasmic chaperones $(5,24,45-47)$ like SurA $(48-52)$ or DegP (53) are needed for OMP folding in vivo, depending on the stress conditions.

Omp A Folds via Parallel Folding Pathways in Vitro. The kinetics of OmpA folding into lipid bilayers was systematically analyzed in a previous study (13), and the rate law of this kinetics was determined from the concentration dependencies using the method of initial rates as described (25). Three independent techniques were used, namely, fluorescence and circular dichroism (CD) spectroscopy and monitoring of the formation of folded OmpA by electrophoresis (13). At pH 10, folding kinetics of OmpA into neutral lipid membranes of different short-chain phosphatidylcholines was in excellent agreement with a single insertion and folding step described by a second-order rate law (25), given by eq 1 in Materials and Methods. At high lipid concentrations, this rate law was well approximated by a pseudofirst-order rate law (25), eq 2; see ref 13 for details. Consequently, for each method, folding kinetics was well described by singleexponential fit functions $(13,54,55)$, eq 3 . However, rate constants obtained by fluorescence spectroscopy were about 4 times greater than those obtained by CD spectroscopy or by SDS-PAGE analysis (13). This indicated that the TMD of OmpA first adsorbed to the polar/apolar interface of the membrane as observed by fluorescence, before the secondary and the tertiary structures of the $\beta$-barrel domain were formed at similar rates.

Here, we analyzed the experimental time courses of OmpA folding by fitting functions corresponding to two different kinetic models. Single-exponential fits, represented as dotted lines in
Figure 1-3 and corresponding to pseudo-first-order kinetics $(13,54,55)$, were in good agreement with the experimental data when urea-unfolded OmpA was folded into pure DOPC bilayers above $\mathrm{pH} 7$ or below $\mathrm{pH} 5.5$ (Figure 3B). They were also in good agreement with the experimentally observed slow folding kinetics of Skp-bound OmpA into DOPC, independent of $\mathrm{pH}$ and the presence of LPS (Figure 3C-F). However, the folding kinetics of OmpA were not well described by single-exponential fits when OmpA was folded into DOPC bilayers in the titration region of OmpA between $\mathrm{pH} 5.5$ and $\mathrm{pH} 7$ (Figure 3A) or when OmpA was folded into bilayers composed of DOPC/DOPE/DOPG (Figures 1 and 2). These OmpA folding kinetics were instead well described by double-exponential functions calculated by fitting eq 6 to the experimental kinetics. The corresponding kinetic model, see Materials and Methods, eq 4, assumes the presence of two major parallel folding phases that are of pseudo first order. The model is established based on two observations: A large number of experiments have indicated that the fully folded form of OmpA strictly corresponds to the $30 \mathrm{kDa}$ form (for a review, see ref 18 ). The $30 \mathrm{kDa}$ band forms at the expense of unfolded or partially folded, membrane-surface-adsorbed forms of OmpA that all migrate at $35 \mathrm{kDa}$ (see refs 12 and 15 ). Since the conversion of the 35 to the $30 \mathrm{kDa}$ band is biphasic and there are also no intermediate bands observed, the last step of OmpA folding has to be composed of two parallel phases in all cases where a single-exponential time course is insufficient to describe the folding kinetics, similar to a previous report on another outer membrane protein, FomA of F. nucleatum (10).

Here, the $\mathrm{pH}$ dependence of OmpA folding into DOPC bilayers (Figure 3) showed biphasic kinetics mostly in the $\mathrm{pH}$ titration region of OmpA that was previously characterized by fluorescence (20), but not at basic or acidic $\mathrm{pH}$, where the assumption of a single phase for the last folding step was often sufficient, consistent with our earlier report (13). The $\mathrm{pH}$ dependence of the kinetics and the coexistence of two different aqueous forms of OmpA, characterized by a different degree of hydrophobic collapse (20), suggest that between $\mathrm{pH} 5.5$ and $\mathrm{pH} 7$ parallel routes of OmpA folding are present after urea dilution. Therefore, at least two different membrane-adsorbed forms could also be expected under these conditions. These intermediates would both fold independently with different rates into the $30 \mathrm{kDa}$ form, as described by eq 4 . We therefore analyzed all kinetics by fitting eq 6 , the integrated form of eq 4 , to all of our experimental data to obtain the rate constants of the slow and fast phases, $k_{\mathrm{s}}$ and $k_{\mathrm{f}}$, and the contribution of the fast phase, $A_{\mathrm{f}}$, to the folding kinetics of OmpA into lipid bilayers. These fit functions are shown as solid lines in Figures 1-4. They described all experimental time courses of our study quite well, regardless of membrane composition, $\mathrm{pH}$, and presence or absence of Skp or both Skp and LPS. While it cannot be completely excluded that there are more than two parallel folding phases, the assumption of two major parallel steps is sufficient to describe all experimental data measured in this study.

OmpA Folds via Parallel Folding Pathways in Vivo. We observed improved folding rates and yields of Skp-bound OmpA with negatively charged membranes at $\mathrm{pH} \geq 6$ (Figure 2). Interestingly, E. coli can grow also at much lower $\mathrm{pH}$ (43), which suggests that other chaperones might assist OmpA folding in the acidic $\mathrm{pH}$ region. Deletion of either the $\operatorname{skp}(I)$ or the $\operatorname{sur} A(3,4)$ gene led to reduced concentrations of OMPs in the OM and was not lethal. Simultaneous depletion of both Skp and SurA in 

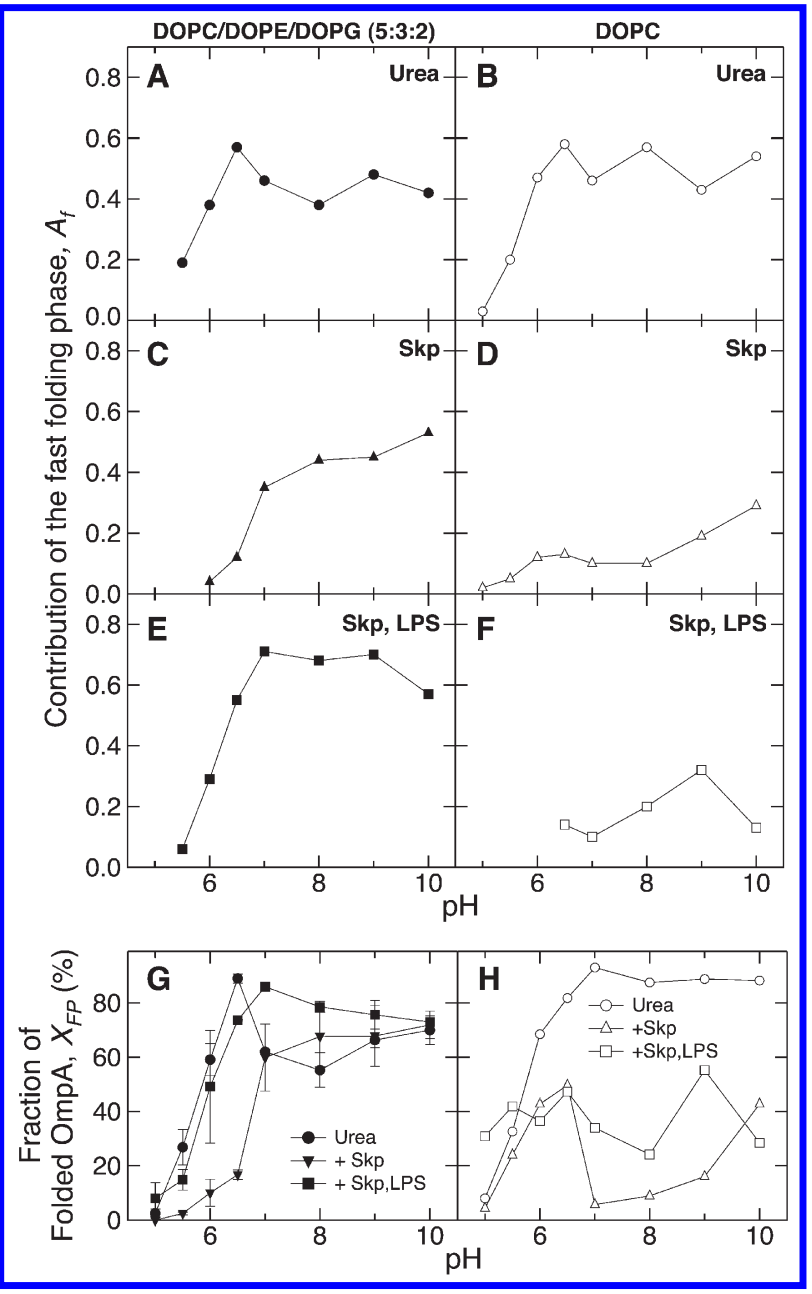

FIgure 5: The analysis of the OmpA folding kinetics indicates that the relative contribution of the fast folding phase, $A_{\mathrm{f}}$, determines the efficiency of OmpA folding. $A_{\mathrm{f}}$ was determined by fitting eq 6 to the experimental data sets shown in Figures $1-3$. The corresponding rate constants $k_{\mathrm{f}}$ and $k_{\mathrm{s}}$ are shown in Figure $\mathrm{S} 3$ of the Supporting Information. (A, C, E) pH dependence of $A_{\mathrm{f}}$ for OmpA folding into bilayers of DOPC/DOPE/DOPG from (A) $8 \mathrm{M}$ urea solution (-) and from the complex with Skp in the absence $(\mathbf{\Delta})(\mathrm{C})$ and in the presence (ם) (E) of LPS. (B, D, F) pH dependence of $A_{\mathrm{f}}$ for OmpA folding into bilayers of DOPC from (B) urea $(O)$ and from the complex with Skp in (D) the absence $(\triangle)$ and in (E) the presence ( $\square$ ) of LPS. (G, H) Experimental yields obtained for OmpA folding into $(\mathrm{G}) \mathrm{DOPC} /$ DOPE/DOPE bilayers or $(\mathrm{H})$ DOPC bilayers from urea $(\mathrm{O})$ and from the complex with Skp in the absence $(\triangle)$ and in the presence ( $\square$ ) of LPS.

$E$. coli had a bacteriostatic effect when cells were grown on rich media, suggesting parallel folding routes in vivo $(6,47)$. This bacteriostatic effect was not observed when cells were grown on minimal media, which was attributed, e.g., to slower growth rates of the cells and the possibility of an alternative slower folding pathway (6). In vivo and when cells are grown on rich media, slow folding forms of OMPs are either degraded by proteases (6) or, alternatively and depending on conditions, their folding might be catalyzed by peptidyl-prolyl cis/trans-isomerases or other chaperones, e.g., SurA, FkpA, or DegP, which are bifunctional periplasmic proteins (see ref 24 for a review). In combination, our present results on parallel folding routes in vitro and the earlier in vivo observations of parallel transport pathways in the periplasm suggest that specific physical properties of the various aqueous forms of OMPs, such as the charge state of the OMP and its compactness, might require chaperones of different properties for optimal OMP folding.
Folding of OmpA Depends on Solubility of the Aqueous Intermediate at Basic pH or on Its Complex Formation with Skp. The fast folding process of urea-unfolded OmpA was promoted at $\mathrm{pH}>\mathrm{p} I_{\mathrm{OmpA}}$, i.e., when OmpA was negatively charged and therefore more soluble, while the slow folding process dominated at a $\mathrm{pH}$ close to $\mathrm{p} I_{\mathrm{OmpA}}$ (Figure $3 \mathrm{~A}, \mathrm{~B}$ ). This was observed independent of the bilayer composition, although kinetics was retarded with negatively charged bilayers (Figure 1) probably due to repulsion of the negatively charged OmpA. The kinetic analysis showed that increased yields of folded OmpA (Figure 5G,H) correlated with an increased relative contribution of the fast folding pathway, $A_{\mathrm{f}}$, for both DOPC/DOPE/DOPG (Figure 5A,C,E) and DOPC bilayers (Figure 5 B,D,F) and not so much with changes in the rate constants $k_{\mathrm{f}}$ and $k_{\mathrm{s}}$ (Figure $\mathrm{S} 3$ of the Supporting Information).

OMPs of $E$. coli generally have a calculated low $\mathrm{p} I$ of $\sim 5-6$ (cf. ref 18), and for OmpA, the difference between basic residues, Arg and Lys $\left(r_{+}=30\right)$, and acidic residues, Asp and Glu $\left(r_{-}=35\right)$ is $\Delta r_{ \pm}=-5$. The charge balance in OmpA is reached by the protonation of its five histidines (His). Typically $\mathrm{p} K_{\mathrm{His}}$ is $\sim 5.5-6$. Four His of OmpA are located in the TMD, and two of them are in close proximity to a negatively charged residue, which can raise $\mathrm{p} K_{\text {His }}$ to $\sim 8(41,42)$. Previous work has shown that the affinity between Skp and OMPs like OmpA and OmpG decreases with increasing ionic strength (20), indicating that binding is strongly affected by electrostatic interactions in addition to hydrophobic interactions. The Skp trimer contains more positively than negatively charged residues, and the difference is $\Delta r_{ \pm}=+15$. Complex formation between OmpA and Skp is observed only at a $\mathrm{pH}$ between $\mathrm{p} I_{\mathrm{OmpA}} \sim 5.5$ and $\mathrm{p} I_{\mathrm{Skp}} \sim 10$. Since OMPs and $\mathrm{Skp}_{3}$ form 1:1 complexes (20), the $\mathrm{Skp}_{3} \cdot \mathrm{OmpA}$ complex has a net positive charge of about +10 between $\mathrm{pH} 6.5$ and $\mathrm{pH}$ 9. This positive net charge of +10 is asymmetrically located in Skp and increases solubility of the Skp. OmpA complex.

Folding of OmpA from the Complexes with the Highly Basic Skp Depends on Interactions of the Positively Charged Skp with Negatively Charged Lipid. Most remarkably, in the presence of Skp or Skp and LPS, folding yields (Figure 5G) and $A_{\mathrm{f}}$ (Figure 5C,E) were strongly increased, when the lipid bilayers contained negatively charged DOPG. Steep time courses were observed for OmpA folding (Figure 2B,D). In contrast, OmpA folding yields (Figure $5 \mathrm{H}$ ) and $A_{\mathrm{f}}$ of OmpA folding (Figure 5D,F) into neutral bilayers of DOPC were poor for Skp-bound OmpA. These time courses displayed slow folding (Figure 3C-F), even when negatively charged LPS was present. A small fraction of Skp-bound OmpA still folded into DOPC bilayers, suggesting that hydrophobic interactions contribute to the release of OmpA from the complex at the membrane/water interface (Figure $3 \mathrm{C}-\mathrm{F}$ ). Since OmpA does not fold in the absence of membranes, whether Skp is present or not (40), folding of OmpA from the complex with Skp is driven by the hydrophobic effect and the formation of a more continuous hydrophobic surface of OmpA at the polar/apolar interface of the membrane. The function of Skp to facilitate OmpA folding at the membrane/water interface of membranes with a negative surface potential is obvious from our raw experimental data and has not yet been demonstrated. It is apparently as important as its chaperone function to prevent misfolding of bound OMPs in solution. Very likely, the negative charges of DOPG attract the positively charged $\mathrm{Skp}_{3} \cdot \mathrm{OmpA}$ complexes and facilitate the release of OmpA from Skp, leading to higher OmpA folding rates. 


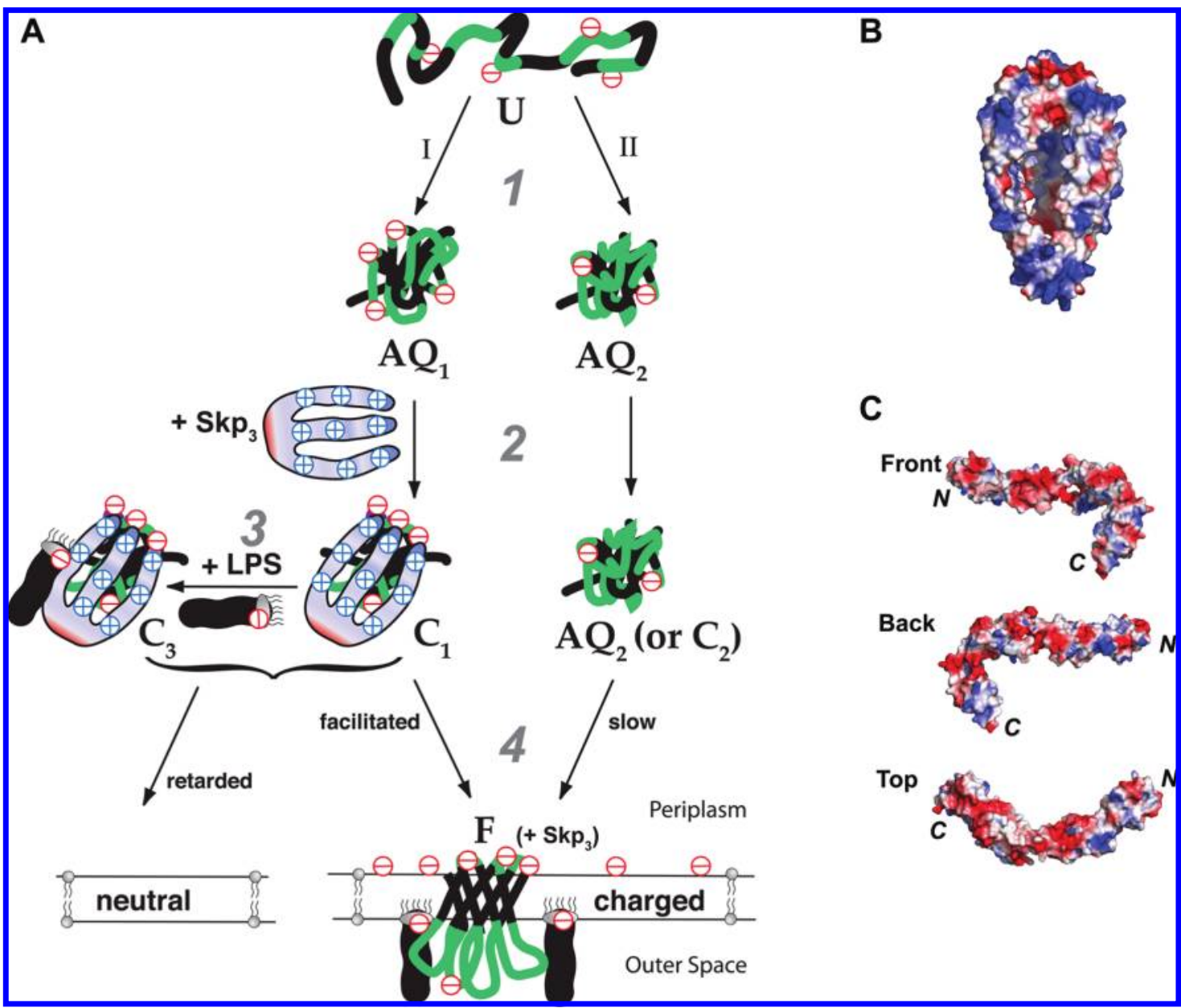

Figure 6: Skp delivers OmpA to the membrane via charge-charge interactions. (A) (1) Unfolded OmpA in $8 \mathrm{M}$ urea (U) is a random coil and negatively charged above its calculated $\mathrm{p} I$ near $\mathrm{pH} \sim 5.5$. Polar segments are colored in green and transmembrane segments in black. For clarity reasons, only the TMD of OmpA is drawn here although we used wild-type OmpA for this study. Upon urea removal, OmpA collapses into more or less compact aqueous forms $\left(\mathrm{AQ}_{1}, \mathrm{AQ}_{2}\right)$ with charged or polar residues on the surface and hydrophobic residues buried inside. Depending on conditions, e.g. $\mathrm{pH}, \mathrm{OmpA}$ can fold in parallel phases into lipid bilayers, which are fast (I) and slow (II). The more compact aqueous OmpA (AQ $)$ folds very slowly. (2) Positively charged $S k p_{3}$ binds $A Q_{1}$ and may also bind to $A Q_{2}$, forming stable complexes $\left(C_{1}, C_{2}\right)$ and preventing further misfolding. (3) $\mathrm{Skp}_{3} \cdot$ OmpA complexes bind negatively charged LPS and form complexes $\mathrm{C}_{3}$. (4) Electrostatic interactions between positive charges of $\mathrm{Skp}_{3}$ of the complexes $\mathrm{C}_{1} / \mathrm{C}_{2} / \mathrm{C}_{3}$ and negatively charged membranes lead to surface binding of the $\mathrm{Skp}_{3} \cdot \mathrm{OmpA}_{\mathrm{m}}$ complexes, followed by OmpA insertion and folding to $\mathrm{F}$ in the membrane. Folding of OmpA from the complexes into membranes without electrostatic surface potential is retarded, leading to reduced yields. (B) Crystal structure of the Skp trimer $(1 \mathrm{sg} 2$ and $1 \mathrm{u} 2 \mathrm{~m})(21,22)$. The trimer forms a large dipole, and the positive residues cover the surface of the Skp tentacles down to their tips. (C) Crystal structure of the periplasmic domain (residues 23-347) of Omp85 (YaeT) (3efc) of E. coli, which is the receptor for unfolded OMPs. The surface of this domain contains several patches of two or more acidic residues, leading to higher local charge densities than caused by the negatively charged lipids at the bilayer surface. The domain extends from the membrane surface into the periplasm and is therefore likely to bind $\mathrm{Skp}_{3} \cdot \mathrm{OMP}$ complexes through electrostatic attraction.

A Mechanism for Skp-Assisted Targeting and Folding of $O M P$ s into a Membrane. The results of this study suggest a mechanism for OMP targeting and folding into membranes that is summarized in the tentative scheme in Figure 6A. After denaturant dilution at neutral and basic $\mathrm{pH}, \mathrm{OmpA}$ forms an aqueous "outside-in" conformation, the "hydrophobically collapsed" intermediate $\mathrm{AQ}_{1}$, in which polar residues of loops and turns form most of the surface (40), shielding the hydrophobic residues of the transmembrane segments from polar solvent (Figure 6A, step 1). OmpA also develops a more compact collapsed conformation $\mathrm{AQ}_{2}$, which dominates at acidic $\mathrm{pH}$ close to $\mathrm{p} I_{\mathrm{OMP}}$ (Figure 6A, step II), because there is less intramolecular charge-charge repulsion. $\mathrm{AQ}_{2}$ requires additional conformational changes for reverting the outside-in orientation of the side chains and therefore folds only very slowly into membranes. In vivo, folding of $\mathrm{AQ}_{2}$ might be facilitated by other chaperones. At an increased net negative charge of OmpA above $\mathrm{p} I_{\mathrm{OmpA}}$, intramolecular side-chain repulsion within OmpA limits the collapse for $\mathrm{AQ}_{1}$, the charged side chains are better hydrated, and aggregation is prevented because of intermolecular repulsion. Therefore, OmpA solubility is greater (35) and $\mathrm{AQ}_{1}$ folds faster into lipid bilayers. When $\mathrm{Skp}_{3}$ is present, $\mathrm{AQ}_{1}$ (and perhaps also $\mathrm{AQ}_{2}$ ) forms stable $\mathrm{Skp}_{3} \cdot$ OmpA complexes, $\mathrm{C}_{1}$ (step 2 ), with nanomolar affinity (20). Negatively charged LPS binds to $\mathrm{Skp}_{3} \cdot$ OmpA complexes (20) to form a ternary complex $\mathrm{C}_{3}$ (step 3). High yields of folded OmpA (F) are obtained for Skp-bound OmpA, i.e., for OmpA bound in complexes $\mathrm{C}_{1}$ and $\mathrm{C}_{3}$, with negatively charged DOPC/DOPE/DOPG bilayers but not with neutral DOPC membranes (step 4). In the absence of negative charges in the membrane, $\mathrm{Skp}_{3}$ retards OMP folding. $\mathrm{OmpA}$ is released, because the negative charges of $\mathrm{PG}$ compete with the negatively charged residues of OmpA for the positive binding sites in Skp. OmpA binding to Skp is therefore weakened at the membrane/water interface. This mechanism favors OmpA insertion and folding into the membrane instead of aggregation of OmpA, since the release of OmpA is triggered 
at the membrane surface rather than in bulk solution. In the additional presence of LPS, release of OmpA may be further facilitated, because the LPS binding sites in Skp, formed by basic residues, Lys and Arg (22), would be screened. This would reduce the affinity of Skp for the negatively charged OmpA as described $(20,40)$.

In the OM, Omp85 is the receptor for unfolded OMPs (56-58). In E. coli, the skp gene follows the gene omp85 (yae $T$ ) immediately downstream on the chromosome, preceding genes encoding enzymes involved in LPS biosynthesis. In our previous study (20), the affinity of Skp for Omp85 from E. coli was up to 2 orders of magnitude greater than for other OMPs, indicating high specificity. In aqueous solution, the soluble $\mathrm{N}$-terminal half of Omp85, which is composed of about 390 residues, likely folds independently of the transmembrane C-terminal domain. Therefore, potential Skp docking sites within the periplasmic domain of Omp85 would be in their native conformation to interact with Skp, explaining the observed high affinity in our previous work. Interestingly, the periplasmic domain of Omp85 has a calculated $\mathrm{p} I$ of 5.35 and a negative net charge of $\Delta r_{ \pm}=-8$. The electrostatic surface potential of its structure (59, 60) (PDB entry 3efc) shows large negatively charged patches composed of two or more acidic residues that may serve as docking sites for Skp (Figure 6C). The previously reported high affinity of the positively charged Skp (Figure 6B) for Omp85 of E. coli (20) and our present study, which demonstrates the role of charge-charge interactions for folding of Skp-bound OMPs into membranes, strongly suggest that the transport of OMPs via Skp across the periplasm could be mediated by interactions between negatively charged residues within OMPs, the positively charged surface of Skp, and the negatively charged surface of the Omp85 periplasmic domain, which protrudes from the OM into the periplasm. We propose that Skp directly transports OMPs from the inner membrane to the OM and specifically to Omp85 via electrostatic interactions. The inhibiting effect observed for membranes containing PE might also explain why the Omp85 complex (BAM complex) is required for OMP assembly in cells. It is possible that the complex recruits $\mathrm{PG}$ to form assembly points for OMP insertion and folding. It could also be that the Omp85 complex directly attracts Skp.OMP complexes and then subsequently mediates OMP insertion.

\section{SUPPORTING INFORMATION AVAILABLE}

The Supporting Information contains the original SDSpolyacrylamide gels in Figures S1 and S2, from which the kinetic data shown in Figures 2 and 3 were obtained. It also includes Figure S3, which indicates the $\mathrm{pH}$ dependence of the rate constants of the fast and slow phases that were obtained by fitting eq 6 to the OmpA folding kinetics shown in Figures 1-3. This material is available free of charge via the Internet at http://pubs.acs.org.

\section{REFERENCES}

1. Chen, R., and Henning, U. (1996) A periplasmic protein (Skp) of Escherichia coli selectively binds a class of outer membrane proteins. Mol. Microbiol. 19, 1287-1294.

2. Schäfer, U., Beck, K., and Müller, M. (1999) Skp, a molecular chaperone of Gram-negative bacteria, is required for the formation of soluble periplasmic intermediates of outer membrane proteins. J. Biol. Chem. 274, 24567-24574.

3. Rouvière, P. E., and Gross, C. A. (1996) SurA, a periplasmic protein with peptidyl-prolyl isomerase activity, participates in the assembly of outer membrane porins. Genes Dev. 10, 3170-3182.
4. Lazar, S. W., and Kolter, R. (1996) SurA assists the folding of Escherichia coli outer membrane proteins. J. Bacteriol. 178, 17701773.

5. Missiakas, D., Betton, J. M., and Raina, S. (1996) New components of protein folding in extracytoplasmic compartments of Escherichia coli SurA, FkpA and Skp/OmpH. Mol. Microbiol. 21, 871-884.

6. Rizzitello, A. E., Harper, J. R., and Silhavy, T. J. (2001) Genetic evidence for parallel pathways of chaperone activity in the periplasm of Escherichia coli. J. Bacteriol. 183, 6794-6800.

7. Buchanan, S. K. (1999) $\beta$-barrel proteins from bacterial outer membranes: structure, function and refolding. Curr. Opin. Struct. Biol. 9 , 455-461

8. Surrey, T., and Jähnig, F. (1992) Refolding and oriented insertion of a membrane protein into a lipid bilayer. Proc. Natl. Acad. Sci. U.S.A 89, 7457-7461.

9. Huysmans, G. H., Radford, S. E., Brockwell, D. J., and Baldwin, S. A. (2007) The N-terminal helix is a post-assembly clamp in the bacterial outer membrane protein PagP. J. Mol. Biol. 373, 529-540.

10. Pocanschi, C. L., Apell, H.-J., Puntervoll, P., Høgh, B. T., Jensen, H. B., Welte, W., and Kleinschmidt, J. (2006) The major outer membrane protein of Fusobacterium nucleatum (FomA) folds and inserts into lipid bilayers via parallel folding pathways. $\underline{\mathrm{J} \text {. Mol. Biol }}$ $355,548-561$.

11. Shanmugavadivu, B., Apell, H. J., Meins, T., Zeth, K., and Kleinschmidt, J. H. (2007) Correct folding of the $\beta$-barrel of the human

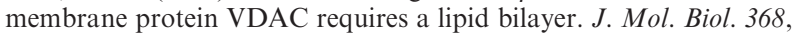
66-78.

12. Kleinschmidt, J. H., and Tamm, L. K. (1996) Folding intermediates of a $\beta$-barrel membrane protein. Kinetic evidence for a multi-step membrane insertion mechanism. Biochemistrv 35, 12993-13000.

13. Kleinschmidt, J. H., and Tamm, L. K. (2002) Secondary and tertiary structure formation of the $\beta$-barrel membrane protein OmpA is synchronized and depends on membrane thickness. J. Mol. Biol. 324, 319-330.

14. Burgess, N. K., Dao, T. P., Stanley, A. M., and Fleming, K. G. (2008) Beta-barrel proteins that reside in the Escherichia coli outer membrane in vivo demonstrate varied folding behavior in vitro. J. Biol. Chem 283, 26748-26758.

15. Kleinschmidt, J. H., and Tamm, L. K. (1999) Time-resolved distance determination by tryptophan fluorescence quenching: probing intermediates in membrane protein folding. Biochemistrv 38, 49965005 .

16. Kleinschmidt, J. H., den Blaauwen, T., Driessen, A., and Tamm, L. K. (1999) Outer membrane protein A of E. coli inserts and folds into lipid bilayers by a concerted mechanism. Biochemistrv 38, 5006-5016.

17. Kleinschmidt, J. H., Wiener, M. C., and Tamm, L. K. (1999) Outer membrane protein A of $E$. coli folds into detergent micelles, but not in the presence of monomeric detergent. Protein Sci. 8, 2065-2071.

18. Kleinschmidt, J. H. (2006) Folding kinetics of the outer membrane proteins OmpA and FomA into phospholipid bilayers. Chem. Phvs. Lipids 141, 30-47.

19. Bulieris, P. V., Behrens, S., Holst, O., and Kleinschmidt, J. H. (2003) Folding and insertion of the outer membrane protein OmpA is assisted by the chaperone Skp and by lipopolysaccharide. J. Biol. Chem. 278, 9092-9099.

20. Qu, J., Mayer, C., Behrens, S., Holst, O., and Kleinschmidt, J. H. (2007) The trimeric periplasmic chaperone Skp of Escherichia coli forms 1:1 complexes with outer membrane proteins via hydrophobic and electrostatic interactions. J. Mol. Biol. 374, 91-105.

21. Korndörfer, I. P., Dommel, M. K., and Skerra, A. (2004) Structure of the periplasmic chaperone Skp suggests functional similarity with cytosolic chaperones despite differing architecture. Nat. Struct. Mol. Biol. 11, 1015-1020.

22. Walton, T. A., and Sousa, M. C. (2004) Crystal structure of Skp, a prefoldin-like chaperone that protects soluble and membrane proteins from aggregation. Mol. Cell 15, 367-374.

23. Schlapschy, M., Dommel, M. K., Hadian, K., Fogarasi, M., Korndörfer, I. P., and Skerra, A. (2004) The periplasmic E. coli chaperone Skp is a trimer in solution: biophysical and preliminary crystallographic characterization. Biol. Chem. 385, 137-143.

24. Kleinschmidt, J. H. (2007) Assembly of integral membrane proteins from the periplasm into the outer membrane, in The Periplasm (Ehrmann, M., Ed.) pp 30-66, ASM Press, Herndon, VA.

25. Atkins, P. W. (1998) Physical Chemistry, 6th ed., W. H. Freeman, New York.

26. Schweizer, M., Hindennach, I., Garten, W., and Henning, U. (1978) Major proteins of the Escherichia coli outer cell envelope membrane. Interaction of protein II with lipopolysaccharide. Eur. J. Biochem. 82, 211-217. 
27. Dornmair, K., Kiefer, H., and Jähnig, F. (1990) Refolding of an integral membrane protein. OmpA of Escherichia coli. J. Biol. Chem. $265,18907-18911$.

28. Rodionova, N. A., Tatulian, S. A., Surrey, T., Jähnig, F., and Tamm, L. K. (1995) Characterization of two membrane-bound forms of OmpA. Biochemistrv 34, 1921-1929.

29. Sugawara, E., Steiert, M., Rouhani, S., and Nikaido, H. (1996) Secondary structure of the outer membrane proteins OmpA of Escherichia coli and OprF of Pseudomonas aeruginosa. J. Bacteriol. $178,6067-6069$.

30. Vogel, H., and Jähnig, F. (1986) Models for the structure of outermembrane proteins of Escherichia coli derived from Raman spectroscopy and prediction methods. J. Mol. Biol. 190, 191-199.

31. Arora, A., Rinehart, D., Szabo, G., and Tamm, L. K. (2000) Refolded outer membrane protein A of Escherichia coli forms ion channels with two conductance states in planar lipid bilayers. J. Biol. Chem. 275, $1594-1600$.

32. Lowry, O. H., Rosebrough, N. J., Farr, A. L., and Randall, R. J. (1951) Protein measurement with the Folin phenol reagent. J. Biol. Chem. 193, 265-275.

33. Vinogradov, E. V., Van Der Drift, K., Thomas-Oates, J. E., Meshkov, S., Brade, H., and Holst, O. (1999) The structures of the carbohydrate backbones of the lipopolysaccharides from Escherichia coli rough mutants F470 (R1 core type) and F576 (R2 core type). Eur. J. Biochem. 261, 629-639.

34. Müller-Loennies, S., Holst, O., and Brade, H. (1994) Chemical structure of the core region of Escherichia coli J-5 lipopolysaccharide. Eur. J. Biochem. 224, 751-760.

35. Surrey, T., and Jähnig, F. (1995) Kinetics of folding and membrane insertion of a $\beta$-barrel membrane protein. J. Biol. Chem. 270, 2819928203.

36. Laemmli, U. K. (1970) Cleavage of structural proteins during the assembly of the head of bacteriophage T4. Nature 227, 680-685.

37. Weber, K., and Osborne, M. (1964) The reliability of molecular weight determinations by dodecyl sulfate-polyacrylamide gel electrophoresis. J. Biol. Chem. 244, 4406-4412.

38. Conlan, S., and Bayley, H. (2003) Folding of a monomeric porin, OmpG, in detergent solution. Biochemistrv 42, 9453-9465.

39. Puntervoll, P., Ruud, M., Bruseth, L. J., Kleivdal, H., Høgh, B. T., Benz, R., and Jensen, H. B. (2002) Structural characterization of the fusobacterial non-specific porin FomA suggests a 14-stranded topology, unlike the classical porins. Microbiology 148, 33953403.

40. Qu, J., Behrens-Kneip, S., Holst, O., and Kleinschmidt, J. H. (2009) Binding regions of outer membrane protein $\mathrm{A}$ in complexes with the periplasmic chaperone Skp. A site-directed fluorescence study. Biochemistrv 48, 4926-4936.

41. Bashford, D., Case, D. A., Dalvit, C., Tennant, L., and Wright, P. E. (1993) Electrostatic calculations of side-chain $\mathrm{p} K_{\mathrm{a}}$ values in myoglobin and comparison with NMR data for histidines. Biochemistry 32, 8045-8056.

42. Jamin, M., Geierstanger, B., and Baldwin, R. L. (2001) The $\mathrm{p} K_{\mathrm{a}}$ of His-24 in the folding transition state of apomyoglobin. Proc. Natl. Acad. Sci. U.S.A. 98, 6127-6131.

43. Stancik, L. M., Stancik, D. M., Schmidt, B., Barnhart, D. M., Yoncheva, Y. N., and Slonczewski, J. L. (2002) pH-dependent expression of periplasmic proteins and amino acid catabolism in Escherichia coli. J. Bacteriol. 184, 4246-4258.

44. Wilks, J. C., and Slonczewski, J. L. (2007) pH of the cytoplasm and periplasm of Escherichia coli: rapid measurement by green fluorescent protein fluorimetry. J. Bacteriol. 189, 5601-5607.

45. Antonoaea, R., Furst, M., Nishiyama, K., and Müller, M. (2008) The periplasmic chaperone PpiD interacts with secretory proteins exiting from the SecYEG translocon. Biochemistrv 47, 5649-5656.

46. Dartigalongue, C., Missiakas, D., and Raina, S. (2001) Characterization of the Escherichia coli $\sigma^{\mathrm{E}}$-regulon. J. Biol. Chem. 276, 2086620875.

47. Sklar, J. G., Wu, T., Kahne, D., and Silhavy, T. J. (2007) Defining the roles of the periplasmic chaperones SurA, Skp, and DegP in Escherichia coli. Genes Dev. 21, 2473-2484.

48. Behrens, S., Maier, R., de Cock, H., Schmid, F. X., and Gross, C. A. (2001) The SurA periplasmic PPIase lacking its parvulin domains functions in vivo and has chaperone activity. EMBO J. 20, 285294.

49. Bitto, E., and McKay, D. B. (2004) Binding of phage-display-selected peptides to the periplasmic chaperone protein SurA mimics binding of unfolded outer membrane proteins. FEBS Lett. 568, 94-98.

50. Bitto, E., and McKay, D. B. (2002) Crystallographic structure of SurA, a molecular chaperone that facilitates folding of outer membrane porins. Structure 10, 1489-1498.

51. Hennecke, G., Nolte, J., Volkmer-Engert, R., Schneider-Mergener, J., and Behrens, S. (2005) The periplasmic chaperone SurA exploits two features characteristic of integral outer membrane proteins for selective substrate recognition. J. Biol. Chem. 280, 2354023548 .

52. Justice, S. S., Lauer, S. R., Hultgren, S. J., and Hunstad, D. A. (2006) Maturation of intracellular Escherichia coli communities requires SurA. Infect. Immun. 74, 4793-4800.

53. Krojer, T., Garrido-Franco, M., Huber, R., Ehrmann, M., and Clausen, T. (2002) Crystal structure of DegP (HtrA) reveals a new protease-chaperone machine. Nature 416, 455-459.

54. Marsh, D., Shanmugavadivu, B., and Kleinschmidt, J. H. (2006) Membrane elastic fluctuations and the insertion and tilt of $\beta$-barrel proteins. Biophvs. J. 91, 227-232.

55. Pocanschi, C. L., Patel, G. J., Marsh, D., and Kleinschmidt, J. H. (2006) Curvature elasticity and refolding of OmpA in large unilamellar vesicles. Biophvs. J. 91, L75-L78.

56. Voulhoux, R., Bos, M. P., Geurtsen, J., Mols, M., and Tommassen, J. (2003) Role of a highly conserved bacterial protein in outer membrane protein assembly. Science (New York) 299, 262-265.

57. Doerrler, W. T., and Raetz, C. R. (2005) Loss of outer membrane proteins without inhibition of lipid export in an Escherichia coli YaeT mutant. J. Biol. Chem. 280, 27679-27687.

58. Gentle, I., Gabriel, K., Beech, P., Waller, R., and Lithgow, T. (2004) The Omp85 family of proteins is essential for outer membrane biogenesis in mitochondria and bacteria. J. Cell Biol. 164, 19-24.

59. Gatzeva-Topalova, P. Z., Walton, T. A., and Sousa, M. C. (2008) Crystal structure of YaeT: conformational flexibility and substrate recognition. Structure 16, 1873-1881.

60. Kim, S., Malinverni, J. C., Sliz, P., Silhavy, T. J., Harrison, S. C., and Kahne, D. (2007) Structure and function of an essential component of the outer membrane protein assembly machine. Science 317, 961-964. 